\title{
Ouantum integrable system with two color components in two dimensions *
}

\author{
Mu-Lin $\operatorname{Yan}^{\dagger}$ \\ Center for Fundamental Physics \\ University of Science and Technology of China \\ Hefei Anhui 230026, P.R.China
}

Dec. 7,2000

\begin{abstract}
The Davey-Stewartson 1(DS1) system[9] is an integrable model in two dimensions. A quantum DS1 system with 2 colour-components in two dimensions has been formulated. This twodimensional problem has been reduced to two one-dimensional many-body problems with 2 colour-components. The solutions of the two-dimensional problem under consideration has been constructed from the resulting problems in one dimensions. For latters with the $\delta$-function interactions and being solved by the Bethe ansatz, we introduce symmetrical and antisymmetrical Young operators of the permutation group and obtain the exact solutions for the quantum DS1 system. The application of the solusions is discussed.
\end{abstract}

PACS numbers: 05.30.-d, 03.65.-w

(This paper has been published in Phys. Rev., E61, 4745 (2000))

\footnotetext{
${ }^{*}$ The work was supported in part by National Fund of China though C N Yang and the Grant LWTZ-1298 of Chinese Academy of Science.

${ }^{\dagger}$ E-mail address: mlyan@ustc.edu.cn
} 


\section{1, Introduction :}

The Davey-Stewartson 1 (DS1) system is an integrable model in space of two spatial and one temporal dimensions $((2+1) \mathrm{D})$. The quantized DS1 system with scalar fields (1 component or shortly 1C) can be formulated in terms of the Hamiltonian of quantum many-body problem in two dimensions, and some of them can be solved exactly[1] [2]. Particularly, it has been shown in ref. 2] that these $2 \mathrm{D}$ quantum $N$-body system with $1 \mathrm{C}$-fields can be reduced to the solvable one-dimensional (1D) quantum $N$-body systems with 1C-fields and with two-body potentials [3]. Thus through solving $1 \mathrm{D}$ quantum $N$-body problems with $1 \mathrm{C}$-fields we can get the solutions for 2D quantum $N$-body problems with $1 \mathrm{C}$-fields. Here the key step is to separate the spatial variables of 2D quantum $N$-body problems with $1 \mathrm{C}$-fields by constructing an ansatz[1][2]

$$
\Psi\left(\xi_{1}, \cdots, \xi_{N}, \eta_{1}, \cdots, \eta_{N}\right)=\prod_{i<j}\left(1-\frac{c}{4} \epsilon\left(\xi_{i j}\right) \epsilon\left(\eta_{i j}\right)\right) X\left(\xi_{1}, \cdots, \xi_{N}\right) Y\left(\eta_{1}, \cdots, \eta_{N}\right)
$$

where $\xi_{i j}=\xi_{i}-\xi_{j}$ and $\eta_{i j}=\eta_{i}-\eta_{j}$. This ansatz will be called the N-body variable-separation ansatz. It is well known that the variable-separation methods are widely used in solving high-dimensional one-particle problems. For instance, for geting the wave functions of electron in hydrgen atom, the ansatz $\Psi(r, \theta, \phi)=R(r) P(\theta) \Phi(\phi)$ is used to reduce the 3D problem to 1D's (this ansatz is what we call the 1-body variable-separation ansatz). The N-body variable-separation ansatz can be thought of as the extension of 1-body variable-separation ansatz. Since the N-body problems are much more complicated than the 1-body problems, it will be highlly nontrivial to construct a N-body variable-separation ansatz. Ref. [1] provided the first example for it and showed that the idea of variable- separating works indeed for the N-body problems induced from the DS1 system .

In this paper, we intend to generalize the above idea to multicomponents DS1 system, namely to construct a new N-body variable-separation ansatz for the multicomponents case and to solve a specific model of 2D quantum DS1 system with multicomponents.

1D N-body model with 2-components has been investigated for long time [4] [5]. The most famous one is the model with delta-function interaction between $2 \mathrm{C}$-fermions 4 . It was solved by the Bethe ansatz[6] and leads to the Yang-Baxter equation and its thermodynamics studies[7] [8] because of the completeness of the Bethe ansatz solutions. In this paper, for definiteness, we shall study specific $2 \mathrm{D}$ quantum $N$-body system with $2 \mathrm{C}$-fields associated with the DS1 system. 
This quantum $N$-body problem under consideration can be reduced to two $1 \mathrm{D}$ quantum $N$-body problems with 2C-fields of ref. [4] and then be exactly solved by using an appropriate N-body variable-separation ansatz and the Bethe ansatz.

\section{2, Quantum DS1 System with Two Components in 2-Dimension :}

Following usual DS1 equation[1] [9], the equation for the DS1 system with two components reads

$$
i \dot{\mathbf{q}}=-\frac{1}{2}\left(\partial_{x}^{2}+\partial_{y}^{2}\right) \mathbf{q}+i A_{1} \mathbf{q}+i A_{2} \mathbf{q}
$$

where $\mathbf{q}$ has two colour components,

$$
\mathbf{q}=\left(\begin{array}{l}
q_{1} \\
q_{2}
\end{array}\right),
$$

and

$$
\begin{aligned}
& \left(\partial_{x}-\partial_{y}\right) A_{1}=-i c\left(\partial_{x}+\partial_{y}\right)\left(\mathbf{q}^{\dagger} \mathbf{q}\right) \\
& \left(\partial_{x}+\partial_{y}\right) A_{2}=i c\left(\partial_{x}-\partial_{y}\right)\left(\mathbf{q}^{\dagger} \mathbf{q}\right)
\end{aligned}
$$

where notation $\dagger$ means the hermitian transposition, and $c$ is the coupling constant. Introducing the coordinates $\xi=x+y, \eta=x-y$, we have

$$
\begin{aligned}
& A_{1}=-i c \partial_{\xi} \partial_{\eta}^{-1}\left(\mathbf{q}^{\dagger} \mathbf{q}\right)-i u_{1}(\xi) \\
& A_{2}=i c \partial_{\eta} \partial_{\xi}^{-1}\left(\mathbf{q}^{\dagger} \mathbf{q}\right)+i u_{2}(\eta)
\end{aligned}
$$

where

$$
\partial_{\eta}^{-1}\left(\mathbf{q}^{\dagger} \mathbf{q}\right)=\frac{1}{2}\left(\int_{-\infty}^{\eta} d \eta^{\prime}-\int_{\eta}^{\infty} d \eta^{\prime}\right) \mathbf{q}^{\dagger}\left(\xi, \eta^{\prime}, t\right) \mathbf{q}\left(\xi, \eta^{\prime}, t\right)
$$

and $u_{1}$ and $u_{2}$ are constants of integration. According to ref.[2], we choose them as

$$
\begin{aligned}
& u_{1}(\xi)=\frac{1}{2} \int d \xi^{\prime} d \eta^{\prime} U_{1}\left(\xi-\xi^{\prime}\right) \mathbf{q}^{\dagger}\left(\xi^{\prime}, \eta^{\prime}, t\right) \mathbf{q}\left(\xi^{\prime}, \eta^{\prime}, t\right) \\
& u_{2}(\eta)=\frac{1}{2} \int d \xi^{\prime} d \eta^{\prime} U_{2}\left(\eta-\eta^{\prime}\right) \mathbf{q}^{\dagger}\left(\xi^{\prime}, \eta^{\prime}, t\right) \mathbf{q}\left(\xi^{\prime}, \eta^{\prime}, t\right) .
\end{aligned}
$$

Thus eq.(1) can be written as

$$
\begin{aligned}
i \dot{\mathbf{q}} & =-\left(\partial_{\xi}^{2}+\partial_{\eta}^{2}\right) \mathbf{q}+c\left[\partial_{\xi} \partial_{\eta}^{-1}\left(\mathbf{q}^{\dagger} \mathbf{q}\right)+\partial_{\eta} \partial_{\xi}^{-1}\left(\mathbf{q}^{\dagger} \mathbf{q}\right)\right] \mathbf{q} \\
& +\frac{1}{2} \int d \xi^{\prime} d \eta^{\prime}\left[U_{1}\left(\xi-\xi^{\prime}\right)++U_{2}\left(\eta-\eta^{\prime}\right)\right]\left(\mathbf{q}^{\dagger \prime} \mathbf{q}^{\prime}\right) \mathbf{q}
\end{aligned}
$$


where $\mathbf{q}^{\prime}=\mathbf{q}\left(\xi^{\prime}, \eta^{\prime}, t\right)$. We quantize the system with the canonical commutation relations

$$
\begin{aligned}
& {\left[q_{a}(\xi, \eta, t), q_{b}^{\dagger}\left(\xi^{\prime}, \eta^{\prime}, t\right)\right]_{ \pm}=2 \delta_{a b} \delta\left(\xi-\xi^{\prime}\right) \delta\left(\eta-\eta^{\prime}\right),} \\
& {\left[q_{a}(\xi, \eta, t), q_{b}\left(\xi^{\prime}, \eta^{\prime}, t\right)\right]_{ \pm}=0 .}
\end{aligned}
$$

where $a, b=1$ or $2,[,]_{+}$and $[,]_{-}$are anticommutator and commutator respectively. Then eq.(8) can be written in the form

$$
\dot{\mathbf{q}}=i[H, \mathbf{q}]
$$

where $H$ is the Hamiltonian of the system

$$
\begin{array}{r}
H=\frac{1}{2} \int d \xi d \eta\left(-\mathbf{q}^{\dagger}\left(\partial_{\xi}^{2}+\partial_{\eta}^{2}\right) \mathbf{q}+\frac{c}{2} \mathbf{q}^{\dagger}\left[\left(\partial_{\xi} \partial_{\eta}^{-1}+\partial_{\eta} \partial_{\xi}^{-1}\right)\left(\mathbf{q}^{\dagger} \mathbf{q}\right)\right] \mathbf{q}\right. \\
\left.+\frac{1}{4} \int d \xi^{\prime} d \eta^{\prime} \mathbf{q}^{\dagger}\left[U_{1}\left(\xi-\xi^{\prime}\right)+U_{2}\left(\eta-\eta^{\prime}\right)\right]\left(\mathbf{q}^{\prime \dagger} \mathbf{q}^{\prime}\right) \mathbf{q}\right)
\end{array}
$$

The $N$-particle eigenvalue problem is

$$
H|\Psi\rangle=E|\Psi\rangle
$$

where

$$
|\Psi\rangle=\int d \xi_{1} d \eta_{1} \ldots d \xi_{N} d \eta_{N} \sum_{a_{1} \ldots a_{N}} \Psi_{a_{1} \ldots a_{N}}\left(\xi_{1} \eta_{1} \ldots \xi_{N} \eta_{N}\right) \mathbf{q}_{a_{1}}^{\dagger}\left(\xi_{1} \eta_{1}\right) \ldots \mathbf{q}_{a_{N}}^{\dagger}\left(\xi_{N} \eta_{N}\right)|0\rangle
$$

The $N$-particle wave function $\Psi_{a_{1} \ldots a_{N}}$ is defined by eq.(14), which satisfies the $N$-body Schrödinger equation

$$
\begin{array}{r}
-\sum_{i}\left(\partial_{\xi_{i}}^{2}+\partial_{\eta_{i}}^{2}\right) \Psi_{a_{1} \ldots a_{N}}+c \sum_{i<j}\left[\epsilon\left(\xi_{i j}\right) \delta^{\prime}\left(\eta_{i j}\right)+\epsilon\left(\eta_{i j}\right) \delta^{\prime}\left(\xi_{i j}\right)\right] \Psi_{a_{1} \ldots a_{N}} \\
+\sum_{i<j}\left[U_{1}\left(\xi_{i j}\right)+U_{2}\left(\eta_{i j}\right)\right] \Psi_{a_{1} \ldots a_{N}}=E \Psi_{a_{1} \ldots a_{N}}
\end{array}
$$

where $\xi_{i j}=\xi_{i}-\xi_{j}, \delta^{\prime}\left(\xi_{i j}\right)=\partial_{\xi_{i}} \delta\left(\xi_{i j}\right)$, and $\epsilon\left(\xi_{i j}\right)=1$ for $\xi_{i j}>0,0$ for $\xi_{i j}=0,-1$ for $\xi_{i j}<0$. Since there are products of distributions in eq.(15), an appropriate regularezation for avoiding uncertainty is necessary. This issue has been discussed in ref.[10]. 
3, Variable Separation of Quantum DS1 with Two Components and Bethe Ansatz :

Our purpose is to solve the $N$-body Schrödinger equation (15). The results in ref.[2] remind us that we can make the following ansatz

$$
\begin{aligned}
\Psi_{a_{1} \ldots a_{N}}= & \quad \sum^{a_{1}^{\prime}} \ldots \prod_{i<j}^{\prime}\left(1-\frac{c}{4} \epsilon\left(\xi_{i j}\right) \epsilon\left(\eta_{i j}\right)\right) \\
& b_{1}^{\prime} \quad \ldots \quad b_{N}^{\prime} \\
& \times \mathcal{M}_{a_{1} \ldots a_{N}, a_{1}^{\prime} \ldots a_{N}^{\prime}} \mathcal{N}_{a_{1} \ldots a_{N}, b_{1}^{\prime} \ldots b_{N}^{\prime}} X_{a_{1}^{\prime} \ldots a_{N}^{\prime}}\left(\xi_{1} \ldots \xi_{N}\right) Y_{b_{1}^{\prime} \ldots b_{N}^{\prime}}\left(\eta_{1} \ldots \eta_{N}\right)
\end{aligned}
$$

where $\mathcal{M}$ and $\mathcal{N}$ are matrices being independent of $\xi$ and $\eta$, and both $X_{a_{1} \ldots a_{N}}\left(\xi_{1} \ldots \xi_{N}\right)$ and $Y_{b_{1} \ldots b_{N}}\left(\eta_{1} \ldots \eta_{N}\right)$ are one-dimensional wave functions of N-bodies. Substituting eq.(16) into eq.(15), we abtian

$$
\begin{aligned}
-\sum_{i} \partial_{\xi_{i}}^{2} X_{a_{1} \ldots a_{N}}+\sum_{i<j} U_{1}\left(\xi_{i j}\right) X_{a_{1} \ldots a_{N}} & =E_{1} X_{a_{1} \ldots a_{N}} \\
-\sum_{i} \partial_{\eta_{i}}^{2} Y_{b_{1} \ldots b_{N}}+\sum_{i<j} U_{2}\left(\eta_{i j}\right) Y_{b_{1} \ldots b_{N}} & =E_{2} Y_{b_{1} \ldots b_{N}}
\end{aligned}
$$

where $U_{1}\left(\xi_{i j}\right)$ and $U_{2}\left(\eta_{i j}\right)$ are two-body potentials, eqs. (17) (18) are one-dimensional $N$-body Schrödinger equations and $E_{1}+E_{2}=E$. Above derivation indicates that the two-dimensional $N$-body Schrödinger equation (15) has been reduced into two one-dimentional $N$-body Schrödinger equations. Namely, the variables in the two-dimentional $N$-body wave function $\Psi_{a_{1} \ldots a_{N}}$ have been separated.

At this stage $\mathcal{M}$ and $\mathcal{N}$ are unknown temporarily. It is expected that for any given pair of exactly solvable 1D N-body problems and the correspondent solutions, we could construct the solutions $\Psi_{A_{1} \ldots A_{N}}$ for $2 \mathrm{D}$ N-body problems eq.(15) through constructing an appropriate $\mathcal{M} \times \mathcal{N}$ matrix. It has been known that the 1D N-body problem in the form of (17) or (18) can be solved exactly for a class of potentials 4 [5] [11]. To illustrate the construction of $\mathcal{M} \times \mathcal{N}$-matrix, we take both potentials in (17) and (18) the delta functions $U_{1}\left(\xi_{i j}\right)=2 g \delta\left(\xi_{i j}\right)$ and $U_{2}\left(\eta_{i j}\right)=2 g \delta\left(\eta_{i j}\right)$ ( $g>0$, the coupling constant). Then eqs.(17) and (18) become

$$
\begin{aligned}
-\sum_{i} \partial_{\xi_{i}}^{2} X_{a_{1} \ldots a_{N}}+2 g \sum_{i<j} \delta\left(\xi_{i j}\right) X_{a_{1} \ldots a_{N}} & =E_{1} X_{a_{1} \ldots a_{N}} \\
-\sum_{i} \partial_{\eta_{i}}^{2} Y_{b_{1} \ldots b_{N}}+2 g \sum_{i<j} \delta\left(\eta_{i j}\right) Y_{b_{1} \ldots b_{N}} & =E_{2} Y_{b_{1} \ldots b_{N}}
\end{aligned}
$$


As $X$ and $Y$ are wave functions of Fermions with two components, denoted by $X^{F}$ and $Y^{F}$, the problem has been solved by Yang long ago[4] (more explicitly, see ref. [12] and ref.[13]). According to the Bethe ansatz, the continual solution of eq.(9) in the region of $0<\xi_{Q_{1}}<\xi_{Q_{2}}<\ldots<\xi_{Q_{N}}<L$ reads

$$
\begin{aligned}
X^{F}= & \sum_{P} \alpha_{P}^{(Q)} \exp \left\{i\left[k_{P_{1}} \xi_{Q_{1}}+\ldots+k_{P_{N}} \xi_{Q_{N}}\right]\right\} \\
= & \alpha_{12 \ldots N}^{(Q)} e^{i\left(k_{1} \xi_{Q_{1}}+k_{2} \xi_{Q_{2}}+\ldots+k_{N} \xi_{Q_{N}}\right)}+\alpha_{21 \ldots N}^{(Q)} e^{i\left(k_{2} \xi_{Q_{1}}+k_{1} \xi_{Q_{2}}+\ldots+k_{N} \xi_{Q_{N}}\right)} \\
& +(N !-2) \text { others terms }
\end{aligned}
$$

where $X^{F} \in\left\{X_{a_{1} \ldots a_{N}}^{F}\right\}, P=\left[P_{1}, P_{2}, \cdots, P_{N}\right]$ and $Q=\left[Q_{1}, Q_{2}, \cdots, Q_{N}\right]$ are two permutations of the integers $1,2, \ldots, N$, and

$$
\begin{aligned}
\alpha_{\ldots i j \ldots}^{(Q)} & =Y_{j i}^{l m} \alpha_{\ldots j i \ldots}^{(Q)}, \\
Y_{j i}^{l m} & =\frac{-i\left(k_{j}-k_{i}\right) P^{l m}+g}{i\left(k_{j}-k_{i}\right)-g}
\end{aligned}
$$

The eigenvalue is given by

$$
E_{1}=k_{1}^{2}+k_{2}^{2}+\ldots+k_{N}^{2}
$$

where $\left\{k_{i}\right\}$ are determined by the Bethe ansatz equations,

$$
\begin{aligned}
& e^{i k_{j} L}=\prod_{\beta=1}^{M} \frac{i\left(k_{j}-\Lambda_{\beta}\right)-g / 2}{i\left(k_{j}-\Lambda_{\beta}\right)+g / 2} \\
& \prod_{j=1}^{N} \frac{i\left(k_{j}-\Lambda_{\alpha}\right)-g / 2}{i\left(k_{j}-\Lambda_{\alpha}\right)+g / 2}=-\prod_{\beta=1}^{M} \frac{i\left(\Lambda_{\alpha}-\Lambda_{\beta}\right)+g}{i\left(\Lambda_{\alpha}-\Lambda_{\beta}\right)-g}
\end{aligned}
$$

with $\alpha=1, \ldots, M, j=1, \ldots, N$. Through exactly same procedures we can get the solution $Y^{F}$ and $E_{2}$ to eq.(20).

As $X$ and $Y$ are Boson's wave-functions, denoted by $X^{B}$ and $Y^{B}$, it is easy to be shown that

$$
\begin{aligned}
X^{B} & =\sum_{P} \beta_{P}^{(Q)} \exp \left\{i\left[k_{P_{1}} \xi_{Q_{1}}+\ldots+k_{P_{N}} \xi_{Q_{N}}\right]\right\} \\
\beta_{\ldots i j \ldots}^{(Q)} & =Z_{j i}^{l m} \beta_{\ldots j i \ldots}^{(Q)} \\
Z_{j i}^{l m} & =\frac{i\left(k_{j}-k_{i}\right) P^{l m}+g}{i\left(k_{j}-k_{i}\right)-g}
\end{aligned}
$$


and the Bethe ansatz equations are as following 13

$$
\begin{gathered}
e^{i k_{j} L}=(-1)^{N+1} \prod_{i=1}^{N} \frac{k_{j}-k_{i}+i g}{k_{j}-k_{i}-i g} \prod_{\beta=1}^{M} \frac{\Lambda_{\beta}-k_{j}+i g / 2}{\Lambda_{\beta}-k_{j}-i g / 2} \\
\prod_{\alpha=1}^{M} \frac{\Lambda_{\beta}-\Lambda_{\alpha}+i g}{\Lambda_{\beta}-\Lambda_{\alpha}-i g}=(-1)^{M+1} \prod_{j=1}^{N} \frac{\Lambda_{\beta}-k_{j}+i g / 2}{\Lambda_{\beta}-k_{j}-i g / 2} .
\end{gathered}
$$

$Y^{B}$ is same as $X^{B}$. It is well known that $X^{F}$ and $Y^{F}\left(X^{B}\right.$ and $\left.Y^{B}\right)$ are antisymmetrical (symmetrecal) as the coordinates and the colour-indeces of the particles interchanges each other simultaneously, instead of the coordinates interchanges each other merely.

\section{4, Young Operator of Permutation Group :}

For permutation group $S_{N}:\left\{e_{i}, i=1, \cdots, N !\right\}$, the totally symmetrical Young operator is

$$
\mathcal{O}_{N}=\sum_{i=1}^{N !} e_{i},
$$

and the totally antisymmetrical Young operator is

$$
\mathcal{A}_{N}=\sum_{i=1}^{N !}(-1)^{P_{i}} e_{i} .
$$

The Young diagram for $\mathcal{O}_{N}$ is \begin{tabular}{|l|l|l|l|l|}
\hline 1 & 2 & 3 & $\cdots$ & $\mathrm{N}$ \\
\hline
\end{tabular} have

$$
\begin{aligned}
& \mathcal{O}_{3}=1+P^{12}+P^{13}+P^{23}+P^{12} P^{23}+P^{23} P^{12} . \\
& \mathcal{A}_{3}=1-P^{12}-P^{13}-P^{23}+P^{12} P^{23}+P^{23} P^{12} .
\end{aligned}
$$

Lemma 1: $\left(\mathcal{O}_{N} X_{F}\right)\left(\xi_{1}, \xi_{2}, \cdots, \xi_{N}\right)$ is antisymmetrical with respect to the coordinate's interchanges of $\left(\xi_{i} \longleftrightarrow \xi_{j}\right)$.

Proof: From the definition of $\mathcal{O}_{N}$ (eq.(32)), we have

$$
\mathcal{O}_{N} P^{a b}=P^{a b} \mathcal{O}_{N}=\mathcal{O}_{N}
$$


To $N=3$ case, for example, the direct calculations show $\mathcal{O}_{3} P^{12}=P^{12} \mathcal{O}=\mathcal{O}_{3}, \mathcal{O}_{3} P^{23}=P^{23} \mathcal{O}=$ $\mathcal{O}_{3}$ and so on. Using eqs.(36) and (23), we have

$$
\mathcal{O}_{N} Y_{i j}^{l m}=(-1) \mathcal{O}_{N}
$$

From eqs.(21) and (23), $X^{F}$ can be written as

$$
\begin{aligned}
X^{F}= & \left\{e^{i\left(k_{1} \xi_{Q_{1}}+k_{2} \xi_{Q_{2}}+\ldots+k_{N} \xi_{Q_{N}}\right)}+Y_{12}^{12} e^{i\left(k_{2} \xi_{Q_{1}}+k_{1} \xi_{Q_{2}}+\ldots+k_{N} \xi_{Q_{N}}\right)}\right. \\
& \left.+Y_{13}^{23} Y_{12}^{12} e^{i\left(k_{2} \xi_{Q_{1}}+k_{3} \xi_{Q_{2}}+k_{1} \xi_{Q_{3}}+\ldots+k_{N} \xi_{Q_{N}}\right)}+(N !-3) \text { other terms }\right\} \alpha_{12 \ldots N}^{(Q)}
\end{aligned}
$$

Using eqs.(37) and (38), we obtain

$$
\begin{array}{r}
\left(\mathcal{O}_{N} X^{F}\right)\left(\xi_{1}, \cdots, \xi_{N}\right)= \\
+e^{i\left(k_{2} \xi_{Q_{1}}+k_{3} \xi_{Q_{2}}+k_{1} \xi_{Q_{3}}+\ldots+k_{N} \xi_{Q_{1}}+k_{2} \xi_{Q_{2}}+\ldots+k_{N} \xi_{Q_{N}}\right)}+e^{i\left(k_{2} \xi_{Q_{1}}+k_{1} \xi_{Q_{2}}+\ldots+k_{N} \xi_{Q_{N}}\right)} \\
=\sum_{P}(-1)^{P} \exp \left\{i\left[k_{P_{1}} \xi_{Q_{1}}+\ldots+k_{P_{N}} \xi_{Q_{N}}\right]\right\}\left(\mathcal{O}_{N} \alpha_{12 \ldots N}^{(Q)}\right) .
\end{array}
$$

Therefore we conclude that $\left(\mathcal{O}_{N} X^{F}\right)\left(\xi_{1}, \cdots, \xi_{N}\right)$ is antisymmetrical with respect to $\left(\xi_{i} \longleftrightarrow \xi_{j}\right)$.

Lemma 2: $\left(\mathcal{A}_{N} X^{B}\right)\left(\xi_{1}, \xi_{2}, \cdots, \xi_{N}\right)$ is antisymmetrical with respect to the coordinate's interchanges of $\left(\xi_{i} \longleftrightarrow \xi_{j}\right)$.

Proof: Noting (see eqs.(33) (29) (27))

$$
\begin{array}{r}
\mathcal{A}_{N} P^{a b}=P^{a b} \mathcal{A}=-\mathcal{A}_{N}, \\
\mathcal{A}_{N} Z_{i j}^{l m}=(-1) \mathcal{A}_{N},
\end{array}
$$

we then have

$$
\left(\mathcal{A}_{N} X^{B}\right)\left(\xi_{1}, \cdots, \xi_{N}\right)=\sum_{P}(-1)^{P} \exp \left\{i\left[k_{P_{1}} \xi_{Q_{1}}+\ldots+k_{P_{N}} \xi_{Q_{N}}\right]\right\}\left(\mathcal{A}_{N} \beta_{12 \ldots N}^{(Q)}\right) .
$$

Then the Lemma is proved.

\section{5, The Solutions of the Problem :}

The ansatz of eq.(16) can be compactly written as

$$
\Psi=\prod_{i<j}\left(1-\frac{c}{4} \epsilon\left(\xi_{i j}\right) \epsilon\left(\eta_{i j}\right)\right)(\mathcal{M} X)(\mathcal{N} Y)
$$


where $(\mathcal{M} X)$ and $(\mathcal{N} Y)$ are required to be antisymmetrical under the interchanges of the coodinate vairables. According to Lemmas 1 and 2, we see that

$$
\mathcal{M}, \mathcal{N}= \begin{cases}\mathcal{O}_{N} & \text { for 1D Fermion } \\ \mathcal{A}_{N} & \text { for 1D Boson. }\end{cases}
$$

As the DS1 fields $q_{a}(\xi \eta)$ in eq.(1) are $(2+1)$ D Bose fields, the commutators ([, $]_{-}$, see (9) and (10)) are used to quantized the system and the $2 \mathrm{D}$ N-body wave functions denoted in $\Psi^{B}$ must be symmetrical under the colour-interchang $\left(a_{i} \longleftrightarrow a_{j}\right)$ and the coordinate-interchange $\left(\left(\xi_{i} \eta_{i}\right) \longleftrightarrow\right.$ $\left.\left(\xi_{j} \eta_{i}\right)\right)$. Namly, the 2D Bose wave functions $\Psi^{B}$ must satisfy that

$$
\left.P^{a_{i} a_{j}} \Psi^{B}\right|_{\xi_{i} \eta_{i} \longleftrightarrow \xi_{j} \eta_{j}}=\Psi^{B} .
$$

As $q_{a}$ are $(2+1)$ D Fermi fields, the anticommutators should be used, and $\Psi^{F}$ must be antisymmetrical under $\left(a_{i} \longleftrightarrow a_{j}\right)$ and $\left(\left(\xi_{i} \eta_{i}\right) \longleftrightarrow\left(\xi_{j} \eta_{i}\right)\right)$. Namly,

$$
\left.P^{a_{i} a_{j}} \Psi^{F}\right|_{\xi_{i} \eta_{i} \longleftrightarrow \xi_{j} \eta_{j}}=-\Psi^{F}
$$

Thus for the 2D Boson case, two solutions of $\Psi^{B}$ can be constructed as following

$$
\begin{aligned}
\Psi_{1}^{B} & =\prod_{i<j}\left(1-\frac{c}{4} \epsilon\left(\xi_{i j}\right) \epsilon\left(\eta_{i j}\right)\right)\left[\mathcal{O}_{\mathcal{N}} X^{F}\left(\xi_{1} \cdots \xi_{N}\right)\right]\left[\mathcal{O}_{\mathcal{N}} Y^{F}\left(\eta_{1} \cdots \eta_{N}\right)\right] \\
\Psi_{2}^{B} & =\prod_{i<j}\left(1-\frac{c}{4} \epsilon\left(\xi_{i j}\right) \epsilon\left(\eta_{i j}\right)\right)\left[\mathcal{A}_{\mathcal{N}} X^{B}\left(\xi_{1} \cdots \xi_{N}\right)\right]\left[\mathcal{A}_{\mathcal{N}} Y^{B}\left(\eta_{1} \cdots \eta_{N}\right)\right] .
\end{aligned}
$$

Using eqs.(36),(39),(40) and (42), we can check eq.(45) diractly. In addition, from the Bethe ansatz equations (25) (26) (30) (31) and $E=E_{1}+E_{2}$, we can see that the eigenvalues of $\Psi_{1}^{B}$ and $\Psi_{2}^{B}$ are different each other generally, i.e., the states corrosponding to $\Psi_{1}^{B}$ and $\Psi_{2}^{B}$ are non-degenerate.

For the 2D Fermion case, the desired results are

$$
\begin{aligned}
& \Psi_{1}^{F}=\prod_{i<j}\left(1-\frac{c}{4} \epsilon\left(\xi_{i j}\right) \epsilon\left(\eta_{i j}\right)\right)\left[\mathcal{O}_{\mathcal{N}} X^{F}\left(\xi_{1} \cdots \xi_{N}\right)\right]\left[\mathcal{A}_{\mathcal{N}} Y^{B}\left(\eta_{1} \cdots \eta_{N}\right)\right] \\
& \Psi_{2}^{F}=\prod_{i<j}\left(1-\frac{c}{4} \epsilon\left(\xi_{i j}\right) \epsilon\left(\eta_{i j}\right)\right)\left[\mathcal{A}_{\mathcal{N}} X^{B}\left(\xi_{1} \cdots \xi_{N}\right)\right]\left[\mathcal{O}_{\mathcal{N}} Y^{F}\left(\eta_{1} \cdots \eta_{N}\right)\right] .
\end{aligned}
$$

Eq.(46) can also be checked diractly. The eigenvalues corresponding to $\Psi^{F}$ are also determined by the Bethe equations and $E=E_{1}+E_{2}$.

It is similar to ref. [2] that we can prove $\Psi_{1,2}^{B}$ and $\Psi_{1,2}^{F}$ shown in above are of the exact solutions of the eq.(15). Thus we conclude that the 2D quantum many-body problem induced from the quantum DS1 system with 2-component has been solved exactly. 


\section{6, The Ground-State Energies of the System :}

In this section, we discuss the ground-state energies of the DS1 system solved in the previous section by using the Bethe ansatz equations (25), (26) and (30), (31). Let the 2D N-body problem reduced from 2D DS1 system with 2 colour (or spin) components has $M$ colours down and $N-M$ colours up. Therefore both $X^{F, B}\left(\xi_{1}, \xi_{2}, \cdots \xi_{N}\right)$ and $Y^{F, B}\left(\eta_{1}, \eta_{2}, \cdots \eta_{N}\right)$ in eqs(47)-(50) are one dimensional $N$-body wave functions with $M$ colours down and $N-M$ colours up. We are interested in the limit that $N, M$ and the length $L$ of the box go to infinity proportionately, i.e., both $N / L=D$ and $M / L=D_{m}$ are finite.

For one dimensional $N$-fermion problem, by the nested Bethe ansatz (or Bethe-Yang ansatz) equations (25) and (26), the corresponding integration equations for the ground state read [4]

$$
\begin{aligned}
2 \pi \sigma_{1} & =-\int_{-B_{1}}^{B_{1}} \frac{2 g \sigma_{1}\left(\Lambda^{\prime}\right) d \Lambda^{\prime}}{g^{2}+\left(\Lambda-\Lambda^{\prime}\right)^{2}}+\int_{-Q_{1}}^{Q_{1}} \frac{4 g \rho_{1}(k) d k}{g^{2}+4(k-\Lambda)^{2}}, \\
2 \pi \rho_{1} & =1+\int_{-B_{1}}^{B_{1}} \frac{4 g \sigma_{1}(\Lambda) d \Lambda}{g^{2}+4(k-\Lambda)^{2}}
\end{aligned}
$$

where $\rho_{1}(k)$ is particle (i.e.,1D fermion) density distribution function of $k$, and $\sigma_{1}(\Lambda)$ is colour-down particle density distribution function of $\Lambda$. Namely, we have

$$
D=\int_{-Q_{1}}^{Q_{1}} \rho_{1}(k) d k, \quad D_{m}=\int_{-B_{1}}^{B_{1}} \sigma_{1}(\Lambda) d \Lambda, \quad E_{1} / N=D^{-1} \int_{-Q_{1}}^{Q_{1}} k^{2} \rho_{1}(k) d k .
$$

For 1D N-boson case, starting from the nested Bethe ansatz equations (30) and (31), similar integration equations for ground state of bosons can be derived (see Appendix). The results are as follows

$$
\begin{aligned}
2 \pi \sigma_{2} & =\int_{-B_{2}}^{B_{2}} \frac{2 g \sigma_{2}\left(\Lambda^{\prime}\right) d \Lambda^{\prime}}{g^{2}+\left(\Lambda-\Lambda^{\prime}\right)^{2}}-\int_{-Q_{2}}^{Q_{2}} \frac{4 g \rho_{2}(k) d k}{g^{2}+4(k-\Lambda)^{2}} \\
2 \pi \rho_{2} & =1-\int_{-B_{2}}^{B_{2}} \frac{4 g \sigma_{2}(\Lambda) d \Lambda}{g^{2}+4(\Lambda-k)^{2}}+\int_{-Q_{2}}^{Q_{2}} \frac{2 g \rho_{2}\left(k^{\prime}\right) d k^{\prime}}{g^{2}+\left(k-k^{\prime}\right)^{2}}
\end{aligned}
$$

where $\rho_{2}(k)$ and $\sigma_{2}(\Lambda)$ are bosonic particle density distribution function of $k$ and its colour-down particle density distribution function of $\Lambda$ respectively, i.e.,

$$
D=\int_{-Q_{2}}^{Q_{2}} \rho_{2}(k) d k, \quad D_{m}=\int_{-B_{2}}^{B_{2}} \sigma_{2}(\Lambda) d \Lambda, \quad E_{2} / N=D^{-1} \int_{-Q_{2}}^{Q_{2}} k^{2} \rho_{2}(k) d k
$$

The everage energies of the 2D DS1 ground states described by $\Psi_{1}^{B}, \Psi_{2}^{B}, \Psi_{1}^{F}$ and $\Psi_{2}^{F}$ (see eqs $(47)-(50))$ are denoted by $E\left(\Psi_{1}^{B}\right), E\left(\Psi_{2}^{B}\right), E\left(\Psi_{1}^{F}\right)$ and $E\left(\Psi_{2}^{F}\right)$ respectively. Then, the everage 
energies per particle for the ground-states are follows

$$
\begin{aligned}
E\left(\Psi_{1}^{B}\right) / N & =2 E_{1} / N=2 D^{-1} \int_{Q_{1}}^{Q_{1}} k^{2} \rho_{1}(k) d k \\
E\left(\Psi_{2}^{B}\right) / N & =2 E_{2} / N=2 D^{-1} \int_{Q_{2}}^{Q_{2}} k^{2} \rho_{2}(k) d k \\
E\left(\Psi_{1}^{F}\right) / N & =\frac{1}{N}\left(E_{1}+E_{2}\right) \\
& =D^{-1}\left(\int_{Q_{1}}^{Q_{1}} k^{2} \rho_{1}(k) d k+\int_{Q_{2}}^{Q_{2}} k^{2} \rho_{2}(k) d k\right) \\
& =\frac{1}{2}\left(E\left(\Psi_{1}^{B}\right)+E\left(\Psi_{2}^{B}\right)\right), \\
E\left(\Psi_{2}^{F}\right) / N & =E\left(\Psi_{1}^{F}\right) / N .
\end{aligned}
$$

From these equations, the follows can been seen: 1, The everage energies per particle for the ground states of this 2-dimensional (2D-) DS1 problem are reduced into the everage energies per particle of 1-dimensional (1D-)many body problems. As $D$ and $D_{m}$ are given, by solving the integration equations $(51)-(56)$, we obtain the $\rho_{1}(k)$ and $\rho_{2}(k)$, and then get the desired results of $E\left(\Psi_{1}^{B}\right) / N$, $E\left(\Psi_{2}^{B}\right) / N, E\left(\Psi_{1}^{F}\right) / N$ and $E\left(\Psi_{2}^{F}\right) / N$. 2, For the two bosonic solutions of the 2D DS1 system with 2 colours (eqs (47) (48)), the everage ground state energies per particle are twice as large as

one of 1D-fermions or 1D-bosons; 3, For the fermion solutions of this 2D-DS1 system, $E\left(\Psi_{1}^{F}\right) / N$ and $E\left(\Psi_{2}^{F}\right) / N$ are sum of 1D-fermion everage energy per particle and 1D-boson's. 4, In general, $E\left(\Psi_{1}^{B}\right) \neq E\left(\Psi_{2}^{B}\right) \neq E\left(\Psi_{1}^{F}\right.$ or 2 $)$. Namely, for same DS1 system, if the statistics of the wave functions (or particles) is different, the corresponding ground-state energies are different. This is remarkable and reflects the statistical effects in the 2D DS1 system.

\section{7, Discussions and Summary :}

Finally, we would like to speculate some further applications of the results presented in this paper to the mathematical physics. Our results may be useful in the following two respects. Firstly, the Bethe ansatz equations (25), (26) for fermion wave functions and (30), (31) for boson's can be solved respectively, even though the equations are systems of transcendental equations for which the roots are not easy to locate. The so-called string hypothesis is used for the analysis and classification of the roots for the Bethe ansatz equations [7][8]. Thus, we could study thier ground state, the excitation and the thermodynamics based on it [7] [8]. Then, the thermodynamical properties of 
the 1D Bose or Fermi gas with $\delta$-function interaction and with two components can be explored. The eqs.(47)-(50) indicate that under the thermodynamical limit the 2D DS1 gases (with two colour components) are classified into 2D Bose gases and 2D Fermi gases. By eqs (47) (48), the 2D Bose gases are composeted of two 1D Fermi gases or 1D Bose gases, and by eqs (49) (50), the 2D Fermi gases are composeted of 1D Fermi gas and 1D Bose gas. Hence, the thermodynamics of 2D DS1 gases with two colour components can be derived exactly. It would be interesting in physics, because this is an interesting and nontrivial example to illustrate coupling (or fusing) of two 1D 2-component gases with $\delta$-function interactin and with different or same statistics. Secondly, the colourless DS1 equation originated in studies of nonlinear phenomena 9. Five years ago, Pang, $\mathrm{Pu}$ and Zhao 14 showed an example that the solutions of the initial-boundary-value problem for the related classical DS1 equation in ref. [15] are consistent with the solutions for the quantum DS1 system with time-dependent applied forces. This indicates that the classical solutions of DS1 equation are corresponding to the classcal limit of the solutions for the quantum DS1 system. This is actully a new method to reveal the solutions of the colourless DS1 equation. To the quantum DS1 system with colour indices studied in this present paper, similar correspondences are expectable. Hence, the structure of the solutions of the quantum DS1-system with colour indices revealed in this paper would be helpful to understand the corresponding classical solutions of DS1 systems with colour. The specific studies on the above speculations would be meaningful, however, they are beyond the scope of this present paper.

To summarize. We formulated the quantum multicomponent DS1 system in terms of the quantum multicomponent many-body Hamiltonain in 2D space. Then we reduced this 2D Hamiltonain to two 1D multicomponent many-body problems. As the potential between two particles with two components in one dimension is $\delta$-function, the Bethe ansatz was used to solve these 1D problems. By using the ansatz of ref.[1] and introducing some useful Young operators, we presented a new N-body variable-separation ansatz for fusing two 1D-solutions to construct 2D wave functions of the quantum many-body problem which is induced from the quantum 2-component DS1 system. There are two types of wave functions: Boson's and Fermion's. Both of them satisfy the 2D many-body Schrödinger equation of the DS1 system exactly. The results have been used to 
study the ground states of the system. Some further applications of the results presented in this paper are speculated and discussed.

\section{Acknowledgement}

The author would like to thank Y.Chen and B.H.Zhao for thier helpful discussions.

\section{References}

[1] G.D.Pang, F.C.Pu and B.H.Zhao, Phys. Rev. Lett. 65, 3227(1990).

[2] M.L.Yan and B.H.Zhao, Phys.Lett. A168, 25(1992).

[3] M.A.Olshanetsy and A.M.Perelomov, Phys.Rep. 94, 313(1983).

[4] C.N.Yang,Phys.Rev.Lett. 19, 1312(1967); Phys.Rev. 168,1920(1968).

[5] F. Calogero, O. Ragnisco and C. Marchioro, Lett. Nuovo Cimento 12, 383(1975)

B. Sutherland, Rocky Mtn. J. Math. 8, 413 (19778)

[6] H.A.Bethe, Z.Phys. 71, 205(1931).

E.H.Lieb and W.Liniger, Phys.Rev.130, 1605(1963).

[7] C.K.Lai, Phys. Rev. Lett. 26, 1472 (1971); Phys. Rev. A8, 2567 (1973).

[8] T.Schlottmann, Int. J. of Mod. Phys. B11, 355 (1997).

[9] A.Davey and K.Stewartson, Proc. R. Soc.A338, 101(1974).

[10] B.H.Zhao, J.of China Univ. Of Sci. \& Tech. 23, 27(1993).

J.F.Colombean,New Gereralized Functions and Multiplication of Distributions, NorthHoland, Amsterdam, 1984.

[11] B. Sutherland and R.A.Römer, Phys.Rev.Lett.,71, 2789(1993).

[12] C.H.Gu and C.N.Yang, Commun.Math.Phys.122, 105(1989).

[13] H.T.Fan, F.C.Pu and B.H. Zhao, Nucl. Phys. B299, 52(1988). 
[14] G.D.Pang, F.C.Pu and B.H.Zhao, J.Phys. A: Math. Gen. 25, L527 (1992).

[15] A.S.Fokas and M.J.Ablowitz, Nonlinear Phenomena (Lecture Notes in Physics 189), 1989, ed K.B.Wolf(Berlin: Springer).

\section{Appendix}

Let us derive eqs (54) and (55) in the text. We start from the Bethe ansatz equations (30) and (31) of 1D bosons with two colour componts. Taking the logarithm of (30) and (31) respectively, we have

$$
\begin{aligned}
& k_{j} L=2 \pi I_{k}-2 \sum_{i=1}^{N} \tan ^{-1} \frac{k_{j}-k_{i}}{g}-2 \sum_{\beta=1}^{M} \tan ^{-1} \frac{2\left(\Lambda_{\beta}-k_{j}\right)}{g} \\
& 2 \sum_{\alpha=1}^{M} \tan ^{-1} \frac{\Lambda_{\beta}-\Lambda_{\alpha}}{g}=2 \pi J_{\Lambda}+2 \sum_{j=1}^{N} \tan ^{-1} \frac{2\left(\Lambda_{\beta}-k_{j}\right)}{g},
\end{aligned}
$$

where (for the case of $N=$ even, $M=$ odd)

$$
\begin{aligned}
\frac{1}{2}+I_{k} & =\text { successive integers from } 1-\frac{1}{2} N \text { to }+\frac{1}{2} N, \\
J_{\Lambda} & =\text { successive integers from }-\frac{1}{2}(M-1) \text { to }+\frac{1}{2}(M-1) .
\end{aligned}
$$

We can now approach the limit $N \longrightarrow \infty, M \longrightarrow \infty, L \longrightarrow \infty$ proportionally, obtaining

$$
\begin{aligned}
& k=2 \pi f_{2}-2 \int_{-Q_{2}}^{Q_{2}} d k^{\prime} \rho_{2}\left(k^{\prime}\right) \tan ^{-1} \frac{\left(k-k^{\prime}\right)}{g}-2 \int_{-B_{2}}^{B_{2}} d \Lambda \sigma_{2}(\Lambda) \tan ^{-1} \frac{2(\Lambda-k)}{g} \\
& 2 \int_{-B_{2}}^{B_{2}} d \Lambda^{\prime} \sigma_{2}\left(\Lambda^{\prime}\right) \tan ^{-1} \frac{\Lambda-\Lambda^{\prime}}{g}=2 \pi h_{2}+2 \int_{-Q_{2}}^{Q_{2}} d k \rho_{2}(k) \tan ^{-1} \frac{2(\Lambda-k)}{g}, \\
& \frac{d h_{2}}{d \Lambda}=\sigma_{2}, \quad \frac{d f_{2}}{d k}=\rho_{2}, \\
& D=\frac{N}{L}=\int_{-Q_{2}}^{Q_{2}} \rho_{2}(k) d k, \quad D_{m}=\frac{M}{L}=\int_{-B_{2}}^{B_{2}} \sigma_{2}(\Lambda) d \Lambda
\end{aligned}
$$

Or, after differentiation,

$$
\begin{aligned}
2 \pi \sigma_{2} & =\int_{-B_{2}}^{B_{2}} \frac{2 g \sigma_{2}\left(\Lambda^{\prime}\right) d \Lambda^{\prime}}{g^{2}+\left(\Lambda-\Lambda^{\prime}\right)^{2}}-\int_{-Q_{2}}^{Q_{2}} \frac{4 g \rho_{2}(k) d k}{g^{2}+4(k-\Lambda)^{2}} \\
2 \pi \rho_{2} & =1-\int_{-B_{2}}^{B_{2}} \frac{4 g \sigma_{2}(\Lambda) d \Lambda}{g^{2}+4(\Lambda-k)^{2}}+\int_{-Q_{2}}^{Q_{2}} \frac{2 g \rho_{2}\left(k^{\prime}\right) d k^{\prime}}{g^{2}+\left(k-k^{\prime}\right)^{2}}
\end{aligned}
$$

which are just eqs (54) and (55). 